\title{
MODE COUPLING THEORY FOR THE CRITICAL DYNAMICS OF DIPOLAR FERROMAGNETS
}

\author{
E. FREY and F. SCHWABL \\ Institut für Theoretische Physik, Physik-Department der Technischen Universität München, \\ D.8046 Garching, FRG
}

\begin{abstract}
The critical dynamics of ferromagnets has been studied by various experimental methods sampling different regions in wave vector space. Nevertheless there seemed to be a list of contradictions between these experiments. Within a mode coupling theory including both short range exchange and long range dipolar interaction we are able to resolve the seemingly contradictory situation.
\end{abstract}

Above $T_{c}$ the critical dynamics of isotropic ferromagnets has been studied by various experimental methods, sampling different regions in wave vector space. These measurements indicate that the critical dynamics of isotropic ferromagnets cannot be explained solely on the basis of the short range exchange interaction. In hyperfine interaction (HFI) experiments on $\mathrm{Ni}$ and $\mathrm{Fe}$ a crossover in the dynamical critical exponent $z$ from $z=5 / 2$ to $z=2$ was observed [1] indicating a crossover to a dynamics with a non conserved order parameter. This is confirmed by electron spin resonance (ESR) and magnetic relaxation experiments [2-6], where one finds a non vanishing Onsager coefficient at zero wave vector. However, the critical exponent deduced from the wave vector dependence of the neutron scattering data right at the critical temperature is $5 / 2$ [7-11]. But nevertheless the data could not be fitted by the Resibois-Piette [12] scaling function for isotropic ferromagnets [7]. Those apparent discrepancies were recently resolved by a mode coupling (MC) theory [13,14], which takes into account the long range dipole-dipole interaction present in all real ferromagnets. In this paper we will shortly review this theory and then concentrate on comparison with ESR, magnetic relaxation and HFI experiments.

The Hamiltonian for a spin system with both short range exchange and long range dipolar interactions is given by

$H=\int \frac{\mathrm{d}^{3} q}{(2 \pi)^{3}}\left[\left(J_{0}+J q^{2}\right) \delta^{i j}+J g \frac{q^{i} q^{j}}{q^{2}}\right] S^{i}(\boldsymbol{q}) S^{j}(-\boldsymbol{q})$,

where we have used the same notation as in ref. [13]. The parameter $g$ characterizes the ratio of dipolar to exchange interactions and is related to the dipolar wave vector $q_{\mathrm{D}}$ by $\sqrt{\mathrm{g}}=q_{\mathrm{D}} a$. The dipolar interaction has several characteristic features. (I) In contrast to the short range exchange interaction the dipole-dipole 
interaction has a long range and therefore dominates the asymptotic critical behavior of ferromagnets. (II) It breaks the symmetry of the spin fluctuations longitudinal and transverse to the wave vector $q$. This implies that the longitudinal static susceptibility remains finite for $q \rightarrow 0$ and $T \rightarrow T_{\mathrm{c}}$. Furthermore this suggests to decompose the spin operator $\boldsymbol{S}(\boldsymbol{q})$ into a longitudinal and two transverse components with respect to the wave vector $\boldsymbol{q} . \boldsymbol{S}(\boldsymbol{q})=S^{\mathrm{L}}(\boldsymbol{q}) \hat{\boldsymbol{q}}+$ $S^{T_{1}}(\boldsymbol{q}) \hat{\boldsymbol{t}}^{1}(\hat{\boldsymbol{q}})+S^{T_{2}}(\boldsymbol{q}) \hat{\boldsymbol{t}}^{2}(\hat{\boldsymbol{q}})$, where the orthonormal set of unit vectors can be found in ref. [13]. (III) The order parameter is no longer conserved as can be inferred from the equations of motion [13], the general structure of which is

$\dot{S}_{q}^{\alpha}=\int \frac{\mathrm{d}^{3} k}{(2 \pi)^{3}} \cdots\left[(\boldsymbol{q}(2 \boldsymbol{k}-\boldsymbol{q})+\cdots g)\left\{S_{q-k}^{\mathrm{T}_{1}}, S_{k}^{\mathrm{L}}\right\}+\cdots\right]$.

The terms proportional to $g$, resulting from the dipolar term in the Hamiltonian remain finite with wave vector $\boldsymbol{q}$ tending to zero, whereas all the other terms vanish in this limit. This reflects the fact that the dipolar forces lead to a relaxational dynamics in the limit of long wavelengths; i.e. the dynamical critical exponent $z$ equals 2 in the asymptotic critical limit. (IV) The presence of a second length scale $q_{\mathrm{D}}^{-1}$ besides the correlation length $\xi$ implies an extension of the dynamical scaling law according to $(\alpha=\mathrm{L}, \mathrm{T})$

$\Phi^{\alpha}(q, g, \omega)=\frac{1}{\Lambda q^{2} \Omega^{\alpha}(x, y)} \phi^{\alpha}\left(x, y, \nu_{\alpha}\right)$

with the scaling variables

$x=\frac{1}{q \xi}, \quad y=\frac{q_{\mathrm{D}}}{q} \quad$ and $\quad \nu_{\alpha}=\frac{\omega}{\Lambda q^{2} \Omega^{\alpha}(x, y)}$

and the characteristic frequencies $\Omega^{\alpha}(x, y)$. Here $\Phi^{\alpha}(q, g, \omega)$ is the half sided Fourier transform of the Kubo relaxation function defined by

$\Phi^{\alpha}(\boldsymbol{q}, g, t)=\mathrm{i} \lim _{\epsilon \rightarrow 0} \int_{t}^{\infty} \mathrm{d} \tau \mathrm{e}^{-\epsilon \tau}\left\langle\left[S^{\alpha}(\boldsymbol{q}, \tau), S^{\alpha}(\boldsymbol{q}, 0)^{\dagger}\right]\right\rangle$

with the normalization $\Phi^{\alpha}(q, g, t=0)=1$; i.e. the spin variables are normalized with respect to the static susceptibilities.

The essential step of the MC is to consider only two mode processes; i.e. after insertion of the equations of motion into the transport coefficients

$\Gamma^{\alpha}(q, g, t)=\frac{1}{\chi^{\alpha}(q, g)} \Phi\left(\dot{S}^{\alpha}(q, t), \dot{S}^{\alpha}(q, 0) ; q, g, t\right)$

one makes a factorisation approximation of the resulting four-point functions. This leads to

$$
\begin{aligned}
\Gamma^{\alpha}(q, g, t)= & 2 J^{2} k_{\mathrm{B}} T \int \frac{\mathrm{d}^{3} k}{(2 \pi)^{3}} \sum_{\beta, \sigma} v_{\beta \sigma}^{\alpha}(k, q, g, \theta)\left(\delta^{\alpha \mathrm{T}}+\delta^{\alpha \mathrm{T}} \delta^{\beta \mathrm{L}} \delta^{\alpha \mathrm{L}}\right) \\
& \times \frac{\chi^{\beta}(k, g) \chi^{\sigma}(|q-k|, g)}{\chi^{\alpha}(q, g)} \Phi^{\beta}(k, g, t) \Phi^{\sigma}(|\boldsymbol{q}-\boldsymbol{k}|, g, t)
\end{aligned}
$$


where the vertex functions $v_{\beta \sigma}^{\alpha}$ for the decay of the mode $\alpha$ into the modes $\beta$ and $\sigma$ can be found in ref. [13].

The MC result for the transport coefficients eq. (6) together with

$$
\frac{\partial}{\partial t} \Phi^{\alpha}(q, g, t)=-\int_{0}^{t} \mathrm{~d} \tau \Gamma^{\alpha}(q, g, t-\tau) \Phi^{\alpha}(q, g, \tau)
$$

constitute a complete set of self consistent equations. These equations were solved at $T_{\mathrm{c}}$ and above $T_{\mathrm{c}}$ recently [14]. If the transport coefficients vary only slowly with $\omega$ one may approximate the relation functions by Lorentzians. This results in a set of coupled integral equations for the Lorentzian line widths $\Gamma^{\alpha}(q, g)=$ $\Gamma^{\alpha}(q, g, \omega=0)$.

$$
\begin{aligned}
\Gamma^{\alpha}(q, g)= & \frac{J^{2} k_{\mathrm{B}} T}{2 \pi^{2} \chi^{\alpha}(q, g)} \int_{-1}^{+1} \mathrm{~d} \eta \int_{0}^{\infty} \mathrm{d} k k^{2} \sum_{\beta, \sigma} v_{\beta \sigma}^{\alpha}(k, q, g, \eta)\left(\delta^{\sigma \mathrm{T}}+\delta^{\alpha \mathrm{T}} \delta^{\beta \mathrm{L}} \delta^{\sigma \mathrm{L}}\right) \\
& \times \frac{\chi^{\beta}(k, g) \chi^{\sigma}(|\boldsymbol{q}-\boldsymbol{k}|, g)}{\Gamma^{\beta}(k, g)+\Gamma^{\sigma}(|\boldsymbol{q}-\boldsymbol{k}|, g)}
\end{aligned}
$$

Using the scaling law

$\Gamma^{\alpha}(q, g)=\Lambda q^{z} \gamma^{\alpha}(x, y)$

these equations were solved in ref [13]. Concerning the critical dynamical exponent one finds for the longitudinal line width a crossover from $z=5 / 2$ in the isotropic critical region to $z=0$ in the dipolar critical region, whereas for the transverse line width the crossover is from $z=5 / 2$ to $z=2$.

In neutron scattering experiments one measures the cross-section for inelastic magnetic scattering. Therefore one is able to measure the dynamical scaling functions as functions of both temperature and wave vector. We have found that precisely at the critical temperature the crossover of the transverse width from $z=5 / 2$ to $z=2$ is shifted with respect to the static crossover to wave vectors smaller by almost one order of magnitude [13]. This explains why up to now this crossover escaped detection by neutron scattering experiments. Concerning the longitudinal width, experiments are still lacking. Our prediction is that the crossover from $z=5 / 2$ to an uncritical behavior $z=0$ should be more pronounced in this case and, in contrast to the transverse width, it occurs in the immediate vicinity of the static crossover, characterized by $q_{\mathrm{D}}$. Hence it should be detectable in the experimentally accessible wave vector region. The experimental results of Mezei $[7,8]$ on $\mathrm{Fe}$ at $T \geqslant T_{\mathrm{c}}$ taken at a series of temperatures show the $(q \xi)^{-1}$-dependence of our theory. For a quantitative comparison with the experiment it may be necessary (i) to reanalyze the experiments including the crossover in the static exponent $\gamma$ (ii) to use the correct theoretical shape [14] for the determination of the line width (iii) to take into account additional relaxation mechanism (uniaxial terms, spin orbit interaction leading to pseudodipolar forces, etc.), which are asymptotically irrelevant but may lead to an enhancement of the line width in the non asymptotic region. 
Table 1

The dipolar wave vector $q_{\mathrm{D}}$, the Curie temperature $T_{\mathrm{c}}$, the experimental non universal constant for the Onsager coefficient $L_{\text {exp }}$ and for the autocorrelation time $H_{\text {exp. }}$.

\begin{tabular}{lcccccc}
\hline & $\mathrm{EuS}$ & $\mathrm{EuO}$ & $\mathrm{CdCr}_{2} \mathrm{~S}_{4}$ & $\mathrm{CdCr}_{2} \mathrm{Se}_{4}$ & $\mathrm{Fe}$ & $\mathrm{Ni}$ \\
\hline$q_{\mathrm{D}}\left[\AA^{-1}\right]$ & 0.24 & 0.147 & 0.0584 & 0.0351 & 0.045 & 0.013 \\
$T_{\mathrm{c}}[\mathrm{K}]$ & 16.6 & 69.7 & 84.4 & 127.8 & 1040 & 631 \\
$L_{\exp }[\mathrm{GHz}]$ & 66 & 21 & 9.0 & 5.5 & - & - \\
$H_{\text {exp }}\left[10^{-13} \mathrm{sec}\right]$ & - & - & - & - & 6.0 & 9.2 \\
\hline
\end{tabular}

In ESR and magnetic relaxation experiments one measures the real and imaginary part of the electronic response function respectively at zero wave vector and determines therefrom the Onsager coefficient. Turning to our MC equations (8) we find that the longitudinal and transverse line widths at zero wave vector are given by

$\Gamma_{0}^{\alpha}(g)=\frac{g^{2} J^{2}}{3 \pi^{2} \chi_{0}^{\alpha}(g)} k_{\mathrm{B}} T \int_{0}^{\infty} \mathrm{d} k k^{2} \frac{\chi^{\mathrm{L}}(k, g) \chi^{\mathrm{T}}(k, g)}{\Gamma^{\mathrm{L}}(k, g)+\Gamma^{\mathrm{T}}(k, g)}$.

As before the temperature dependence of $\Gamma_{0}^{\alpha}(g)$ entering via $\xi$ is not indicated explicitly. Introducing polar coordinates $\left(r=\sqrt{x^{2}+y^{2}}, y / x=\tan \varphi\right)$ and using the scaling properties of the static and dynamic quantities this coefficient can be written as

$\Gamma_{0}^{\alpha}(g)=\frac{B F\left(g \xi^{2}\right)}{\hat{\chi}_{0}^{\alpha}\left(g \xi^{2}\right)} \equiv B \gamma_{0}\left(g \xi^{2}\right)$

The universal crossover function $F$ on the Onsager coefficient following from eq. (11) is

$F\left(g \xi^{2}\right)=\left(1+\frac{1}{g \xi^{2}}\right)^{-7 / 4} \int_{0}^{\infty} \mathrm{d} r r^{5 / 2} \frac{\hat{\chi}^{\mathrm{L}}(r, \varphi) \hat{\chi}^{\mathrm{T}}(r, \varphi)}{\gamma^{\mathrm{L}}(r, \varphi)+\gamma^{\mathrm{T}}(r, \varphi)}$

and the scaling functions $\hat{\chi}_{0}^{\alpha}\left(g \xi^{2}\right)$ for the susceptibilities at zero wave vector are given by

$\hat{\chi}_{0}^{\alpha}\left(g \xi^{2}\right)=\left\{\begin{array}{ll}\frac{1}{1+\left(g \xi^{2}\right)^{-1}} & \text { for } \alpha=L \\ g \xi^{2} & \text { for } \alpha=T\end{array}\right.$.

Finally $B$ denotes a non universal constant $B=\frac{4}{3} \pi^{2} \Lambda q_{\mathrm{D}}^{5 / 2}$. Because the Onsager coefficient $\Gamma^{\alpha} \chi^{\alpha}$ does not depend on the sample shape and is the same for the transverse and the longitudinal mode this quantity is the most convenient for a comparison with experiment. The universal crossover function $F\left(g \xi^{2}\right)$ is plotted in fig. 1 in units of its value at criticality $F(\infty)$ versus $1 / q_{D} \xi$. For temperatures 


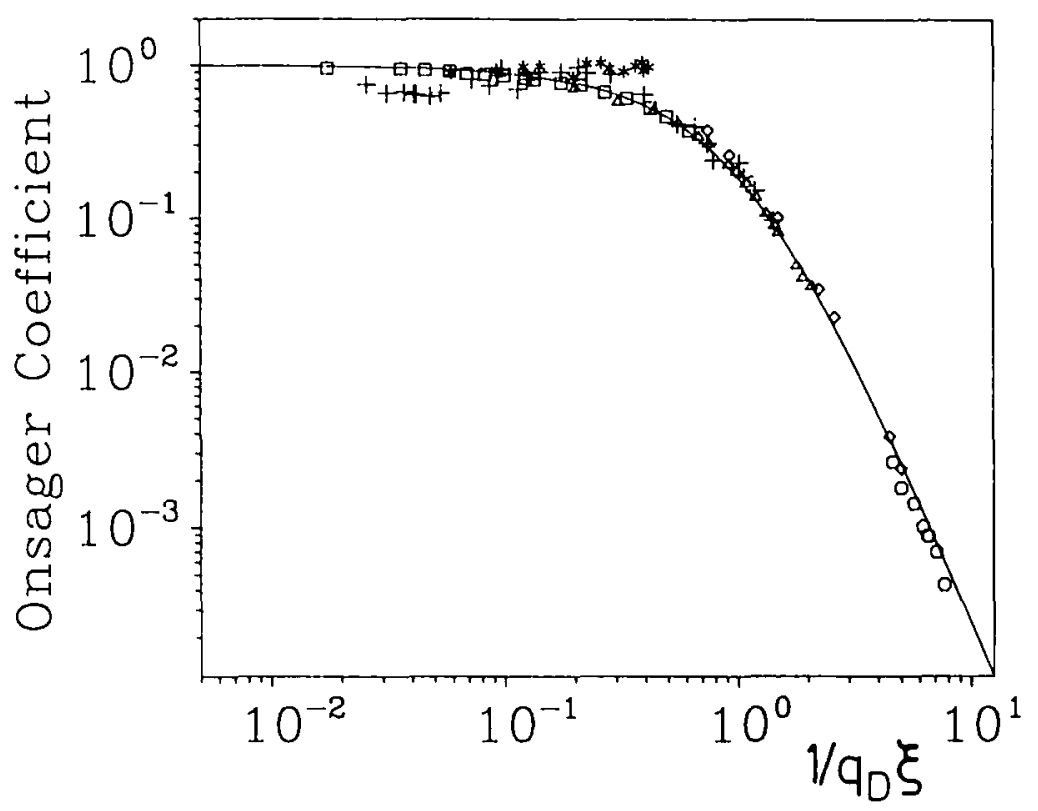

Fig. 1. Universal crossover function $F\left(g \xi^{2}\right)$ for the Onsager coefficient at zero wave vector versus the scaling variable $1 / q_{D} \xi$ (solid line) in units of its value at criticality $F(\infty)$ ). Experimental results for the Onsager coefficient in units of $L_{\text {exp }}$ for EuS ( $\square$, ref. [3]), EuO ( + , ref. [6] and *, ref. [2]), $\mathrm{CdCr}_{2} \mathrm{~S}_{4}(\triangle(\gamma B=1.004 \mathrm{GHz})$, ref. [5] $)$ and $\mathrm{CdCr}_{2} \mathrm{Se}_{4}(\diamond(\gamma B=0.698 \mathrm{GHz})$ and $\circ(\gamma B=9.581$ $\mathrm{GHz}$ ), ref. [4]).

larger than the dipolar crossover temperature $F$ shows a $\xi^{7 / 2}$ behavior and approaches a constant in the strong dipolar limit; i.e. very close to $T_{\mathrm{c}}$. The latter reflects the non conserved nature of the order parameter due to the presence of the dipolar interaction.

The data points in fig. 1 are results from magnetic relaxation experiments $[2,3,6]$ and ESR experiments [2-5] for the Onsager coefficient in EuS, EuO, $\mathrm{CdCr}_{2} \mathrm{~S}_{4}$ and $\mathrm{CdCr}_{2} \mathrm{Se}_{4}$ normalized with respect to their values at criticality $L_{\text {exp }}$. Because ESR experiments are done in magnetic field $B$ there should be an effect on the relaxation rate starting at $\gamma B=\Gamma\left(q=\xi^{-1}\right)[15]$. Those data points are not given in fig. 1 . We note that there is no adjustable parameter for the scaling variable $1 / q_{\mathrm{D}} \xi$. Being normalized with respect to their non universal frequency scale $L_{\exp }$ all experimental data join the theoretical curve. This shows nicely universality concerning the above substances and gives a strong confirmation of our MC theory.

In HFI experiments one observes the nuclear relaxation rate due to the surrounding fluctuating electronic magnetic moments. The standard experiments are performed in the motional narrowing regime [1]. Then assuming a contact interaction $H(t)=A_{\text {contact }} I S(t)$ between $I$ the nuclear and $S$ the electronic 
spins, the nuclear relaxation rate $\tau_{\mathrm{R}}^{-1}$ is directly proportional to the (averaged) spin autocorrelation time $\tau_{\mathrm{c}}$

$\tau_{\mathrm{c}}=\frac{1}{2} \int_{-\infty}^{+\infty} \mathrm{d} t \frac{1}{3} \sum_{\alpha} G^{\alpha \alpha}(\boldsymbol{r}=0, t)$,

where $G^{\alpha \alpha}(r, t)=\frac{1}{2}\left\langle\left\{S^{\alpha}(r, t), S^{\alpha}(0,0)\right\}\right\rangle$ is the spin autocorrelation function. With

$G^{\alpha \alpha}(\boldsymbol{r}, t)=\frac{1}{V_{q}} \int_{B Z} \mathrm{~d}^{3} \boldsymbol{q} \mathrm{e}^{\mathrm{i} q \boldsymbol{r}} \int_{-\infty}^{+\infty} \mathrm{d} \omega \mathrm{e}^{\mathrm{i} \omega t} G^{\alpha \alpha}(\boldsymbol{q}, \omega)$

and the fluctuation dissipation theorem, which in the special case $\omega=0$ reduces to

$G^{\alpha \alpha}(q, \omega=0)=2 k_{\mathrm{B}} T \frac{\chi^{\alpha}(q, g)}{\Gamma^{\alpha}(q, g)}$,

we find for the autocorrelation time

$\tau_{\mathrm{c}}=\frac{k_{\mathrm{B}} T}{V_{q}} \int_{B Z} \mathrm{~d}^{3} q \frac{1}{3} \sum_{\alpha} \frac{\chi^{\alpha}(q, g)}{\Gamma^{\alpha}(q, g)}$.

The $q$-integration extends over the Brillouin zone (BZ), the volume of which is $V_{q}$. Using the static and dynamic scaling laws eq. (15) can be written as

$\tau_{\mathrm{c}} \propto 4 \pi \int \mathrm{d} k k^{-z} \frac{1}{3} \sum_{\alpha} \frac{\hat{\chi}^{\alpha}\left(k \xi, \frac{k}{\sqrt{g}}\right)}{\gamma^{\alpha}\left(k \xi, \frac{k}{\sqrt{g}}\right)}$.

If there were no dipolar interaction, one could extract the temperature dependence from the integral in eq. (16) with the result $\tau_{c} \propto \xi^{z-1}$. We use this expression to define an effective dynamical exponent $z_{\text {eff }}(\tau)$, which depends on the reduced temperature $\tau$ by $\tau_{\mathrm{c}} \propto \xi^{z_{\mathrm{ctI}}-1}$. In the presence of dipolar forces one finds after introducing polar coordinates

$\tau_{\mathrm{c}}=H\left(1+\frac{1}{g \xi^{2}}\right)^{(1-z) / 2} \int_{r_{0}}^{\infty} \mathrm{d} r r^{z-2} \frac{1}{3} \sum_{\alpha} \frac{\hat{\chi}^{\alpha}(r, \varphi)}{\gamma^{\alpha}(r, \varphi)}$,

where $z=5 / 2$ and the non universal constant $H$ is given by

$H=\frac{\left(k_{\mathrm{B}} T\right)^{2}}{32 \pi^{6}\left(\Lambda a^{-5 / 2}\right)^{3}}\left(q_{\mathrm{D}} a\right)^{-3 / 2}$.

The lower cutoff $r_{0}$ is

$r_{0}=\frac{q_{\mathrm{D}}}{q_{\mathrm{Bz}}} \sqrt{1+\frac{1}{g \xi^{2}}}$ 


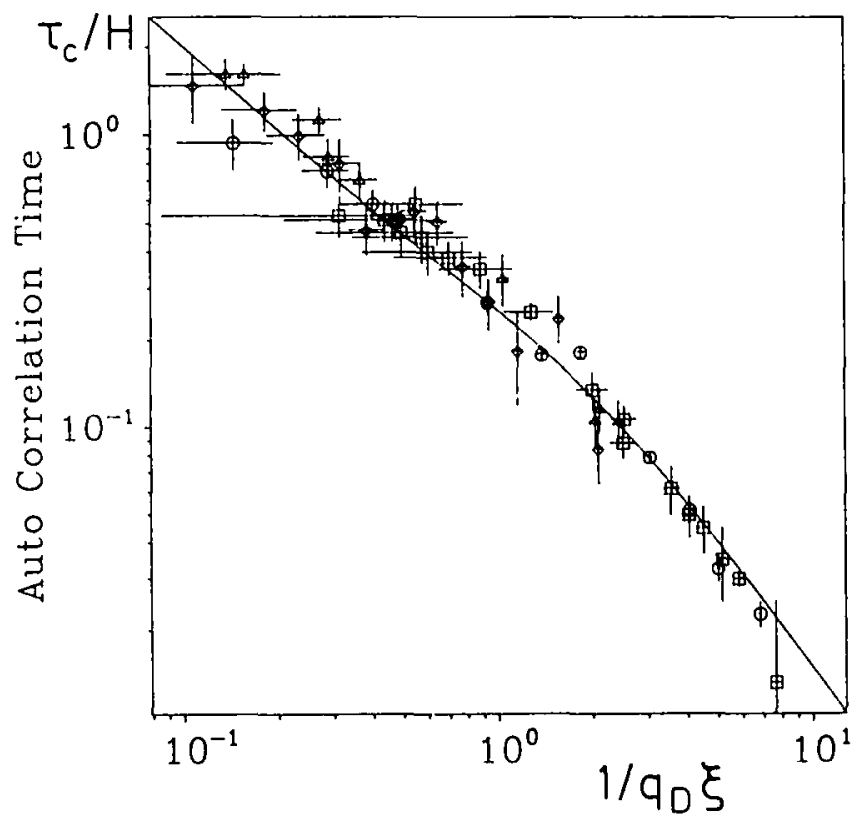

Fig. 2. Auto correlation time $\tau_{\mathrm{c}} / H$ (in units of the non universal constant $H$ ) versus the scaling variable $1 / q_{\mathrm{D}} \xi$ (solid line). Experimental results for the auto correlation time in units of $H_{\exp }$ for Fe (ref. [1]: $\square$ ) and Ni (ref. [16]: $\diamond$, ref. [17]: $\Delta$, ref. [1]: 0 ).

where $q_{\mathrm{Bz}}$ is the boundary of the Brillouin zone. In the critical region it can be neglected $\left(r_{0}=0\right)$, since $q_{\mathrm{BZ}} \gg q_{\mathrm{D}}$ and the integrand in eq. (17) is proportional to $\sqrt{r}$ for small $r$. For very small $\xi$ (outside the critical region) the cutoff reduces the autocorrelation time with respect to the critical value.

The autocorrelation time $\tau_{\mathrm{c}}$ is shown in fig. 2 in units of the non universal constant $H$ (see table 1 ) versus the scaling variable $1 / q_{\mathrm{D}} \xi$. In the asymptotic dipolar region $\tau_{\mathrm{c}}$ diverges like $\tau_{\mathrm{c}} \propto \xi$ corresponding to an effective dynamic exponent $z_{\text {eff }}=2$. By leaving the dipolar critical region there is a crossover to the isotropic Heisenberg region, where $\tau_{\mathrm{c}}$ is characterized by another power law $\tau_{\mathrm{c}} \propto \xi^{3 / 2}$ corresponding to $z_{\text {eff }}=5 / 2$. The data points in Fig. 2 are results of HFI experiments on $\mathrm{Fe}$ and $\mathrm{Ni}[1,16,17]$ for the autocorrelation time $\tau_{\mathrm{c}}$ (in units of their non universal frequency scale $H$ ). As before there is no fit parameter for the scaling variable $1 / q_{\mathrm{D}} \xi$. So we conclude that our MC theory accounts well for the experimental data demonstrating the universal crossover behavior from $z_{\text {eff }}=5 / 2$ to $z_{\text {eff }}=2$ as the critical temperature is approached.

In conclusion we may say that the available experimental data for ferromagnets above $T_{\mathrm{c}}$ are in quantitative agreement with our MC theory. This demonstrates that long range dipolar forces are of great importance for the critical dynamics of real ferromagnets. 


\section{Acknowledgement}

This work has been supported by the German Federal Ministry for Research and Technology (BMFT) under contract number 03-SC1TUM-0.

\section{References}

[1] C. Hohenemser, L. Chow and R.M. Suter, Phys. Rev. B26 (1982) 5056.

[2] J. Kötzler, W. Scheithe, R. Blickhan and E. Kaldis, Solid State Comm. 26 (1978) 641.

[3] J. Kötzler, G. Kamleiter and G. Weber, J. Phys. C9 (1976) L361.

[4] J. Kötzler and H. von Philipsborn, Phys. Rev. Lett. 40 (1978) 790.

[5] J. Kötzler and W. Scheithe, J. of Magn. Magn. Mat. 9 (1978) 4.

[6] R.A. Dunlap and A.M. Gotlieb, Phys. Rev. B22 (1980) 3422.

[7] F. Mezei, Phys. Rev. Lett. 49 (1982) 1096.

[8] F. Mezei, J. of Magn. Magn. Mat. 45 (1984) 67.

[9] F. Mezei, Physica B136 (1986) 417.

[10] P. Böni and G. Shirane, Phys. Rev. B33 (1986) 3012.

[11] P. Böni, G. Shirane, H.G. Bohn and W. Zinn. J. Appl. Phys. 61 (1987) 8.

[12] P. Resibois and C. Piette, Phys. Rev. Lett. 24 (1970) 514.

[13] E. Frey and F. Schwabl, Phys. Lett. A 123 (1987) 49; Z. Phys. B 71 (1988) 355.

[14] E. Frey, F. Schwabl and S. Thoma, Phys. Lett. A 129 (1988) 343, and to be published.

[15] K. Kawasaki, in: Phase Transitions and Critical Phenomena, eds. C. Domb and M.S. Green, Vol. Sa (Academic Press, New York, 1976).

[16] R.C. Reno and C. Hohenemser, Proc. 7th Ann. Conf. on Magnetism and Magnetic Materials, ed. D.C. Graham and J.J. Rhyne (AIP, New York, 1972).

[17] A.M. Gotlieb and C. Hohenemser, Phys. Rev. Lett. 31 (1973) 1222. 